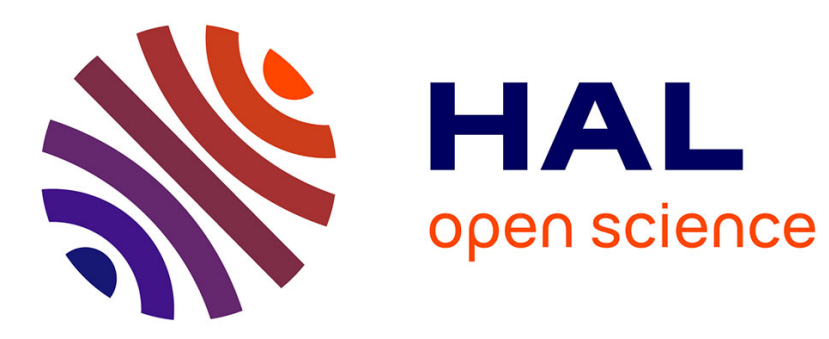

\title{
Les métamorphoses du droit face aux exigences de la transition écologique
}

\author{
Kathia Martin-Chenut, Camila Perruso, Kathia Par
}

\section{To cite this version:}

Kathia Martin-Chenut, Camila Perruso, Kathia Par. Les métamorphoses du droit face aux exigences de la transition écologique. Annales des mines - Série Responsabilité et environnement, 2021, L'enseignement et la formation dans la transition écologique et sociétale, 101, pp.41-43. hal-03357417

\section{HAL Id: hal-03357417 https://hal.science/hal-03357417}

Submitted on 28 Sep 2021

HAL is a multi-disciplinary open access archive for the deposit and dissemination of scientific research documents, whether they are published or not. The documents may come from teaching and research institutions in France or abroad, or from public or private research centers.
L'archive ouverte pluridisciplinaire HAL, est destinée au dépôt et à la diffusion de documents scientifiques de niveau recherche, publiés ou non, émanant des établissements d'enseignement et de recherche français ou étrangers, des laboratoires publics ou privés. 


\title{
Les métamorphoses du droit face aux exigences de la transition écologique
}

\author{
Par Kathia MARTIN-CHENUT \\ Directrice de recherche en droit au CNRS, Institut de sciences juridiques et \\ philosophiques de la Sorbonne (UMR 8103 ISJPS - CNRS/Université Paris1) \\ Et Camila PERRUSO \\ Post-doctorante à l'Institut des sciences philosophiques et juridiques de la Sorbonne
}

La crise écologique bouscule le droit et le force à se réinventer. Le droit participe à la transition écologique, dans la mesure où il permet, d'une part, d'organiser les relations sociales qui en découlent et, d'autre part, d'orienter les transformations nécessaires lorsqu'il s'approprie les normes et valeurs sociales. II peut ainsi jouer un rôle essentiel non seulement a posteriori, mais également tout au long de la transition écologique. Mais celle-ci est complexe, comme les métamorphoses que doit subir le droit pour l'accompagner. Cette contribution met en lumière certaines des adaptations que connaissent certaines catégories juridiques classiques qui nous semblent être bousculées par les exigences d'une transition écologique et solidaire et s'avérer propices à contribuer à sa concrétisation : la souveraineté, la propriété, la personnalité et la responsabilité.

Tout comme d'autres disciplines, le droit est bousculé et se réinvente face à la crise écologique. Celle-ci inscrit notre époque dans une nouvelle ère qui appelle une rupture avec le modèle de développement axé sur la croissance illimitée pour se tourner vers un mode de vie plus durable, en tissant des liens entre tous les peuples, les générations futures et le vivant en général. Or, dans ce monde globalisé où la capacité d'action des États a décliné face à la montée en puissance des forces du marché, lui faisant subir la concurrence de multiples normativités, l'instrumentalisant, voire le marginalisant ${ }^{1}$, le droit retrouve peut-être avec les exigences de la transition écologique une opportunité pour se renouveler.

La problématique écologique ne connaissant pas de frontière, les relations internationales, le droit international et leurs institutions doivent être fondamentalement réorientés pour prendre effectivement en compte la protection de l'environnement et de la planète. Plusieurs changements ont déjà vu le jour sous l'impulsion des grands sommets environnementaux. Depuis les années 1970, des normes à portée variable ont émergé dans la sphère internationale, donnant naissance au droit international de l'environnement, réunissant traités, déclarations et autres instruments normatifs. Plus largement, la crise pousse à l'intégration de la question écologique au sein de différentes branches du droit, en favorisant un verdissement de celui-ci ${ }^{2}$. Pour répondre au risque de «fragmentation du droit

\footnotetext{
${ }^{1}$ Voir OST F. (2020), «Le droit malgré tout », Délibérée, La Découverte, n9, pp. 6-11.

${ }^{2}$ SANDS P. (dir.) (1993), Greening International Law, Earthscan.
} 
international $»^{3}$, le principe d'intégration juridique devrait être davantage mobilisé ${ }^{4}$. Celui-ci se traduit par le renforcement de l'intégration de l'environnement dans toutes sortes de normes juridiques. Il requiert également, lors de l'interprétation des normes, la prise en compte du facteur écologique dans l'arbitrage de différents intérêts. Cela ne signifie pas que les considérations écologiques priment au regard d'autres intérêts, mais qu'il y existe au moins la volonté de les prendre en compte sur un même pied d'égalité.

Ce phénomène trouve également écho en droit français, qui s'est vu s'enrichir d'un corpus juridique en matière environnementale. Si dans ses prémices ce champ du droit concernait surtout des aspects administratifs liés à la gestion de la pollution et des troubles du voisinage ${ }^{5}$, il est aujourd'hui considéré comme un champ fédérateur ${ }^{6}$, dont l'objet doit être pris en compte par les différentes branches du droit et par les décideurs publics. Le rôle de la Charte de l'environnement de 2005, qui est adossée à la Constitution, participe incontestablement à cette intégration de la protection de l'environnement dans l'ordre juridique français ${ }^{7}$. Par ailleurs, un ministère dédié à la Transition écologique [et sociale] est venu succéder à celui de l'Environnement en 2017. N'est-ce pas un signal de la volonté politique (et juridique) d'avancer sur le chemin d'une protection plus globalisante et efficace de l'environnement? Le droit a un rôle essentiel à jouer, non seulement a posteriori, mais tout au long de cette " grande transition $»^{8}$.

Le droit participe à la transition écologique, dans la mesure où il permet, d'une part, d'organiser les relations sociales qui en découlent et, d'autre part, d'orienter les transformations nécessaires lorsqu'il s'approprie les normes et valeurs sociales ${ }^{9}$. Certes, le processus de transition n'est pas linéaire, et les métamorphoses du droit face aux exigences écologiques sont complexes. Dans cet article, nous nous efforcerons de mettre en lumière des adaptations que connaissent certaines catégories juridiques classiques qui nous semblent être bousculées par les exigences d'une transition écologique et solidaire et s'avérer propices à contribuer à sa concrétisation : la souveraineté, la propriété, la personnalité et la responsabilité.

\section{La souveraineté}

Depuis la Modernité, l'ordre juridique international s'est bâti autour de la souveraineté des États. La souveraineté implique la négation de toute subordination des États, en dehors des limitations librement acceptées par eux. L'avènement de

\footnotetext{
${ }^{3}$ « Fragmentation du droit international : difficultés découlant de la diversification et de l'expansion du droit international », rapport du Groupe d'étude de la Commission du droit international (CDI), établi sous sa forme définitive par Martti Koskenniemi (2006), A/CN.4/L.682.

${ }^{4}$ International Law Association, Committee on International Law and Sustainable Development (2006), Report of Toronto's Conference, disponible à : https://www.ila-hq.org/index.php/committees.

${ }^{5}$ UNTERMAIER J. (2005), "La Charte de l'environnement face au droit administratif », Revue juridique de l'Environnement.

${ }^{6}$ MORAND-DEVILLER J. (2019), Le droit de l'environnement, Collection « Que sais-je », Paris, PUF.

${ }^{7}$ COHENDET M.-A. (2019), Droit constitutionnel de l'environnement - Regards croisés, Mare et Martin.

${ }^{8}$ RENOUARD C., BEAU R., GOUPIL C. \& KOENIG C. (dir.) (2020), Manuel de la Grande Transition, Paris, Les Liens qui libèrent.

${ }^{9}$ OST F. (2016), À quoi sert le droit ? Usages, fonctions et finalités, Bruxelles, Bruylant.
} 
l'ONU en 1945 a favorisé le passage d'un droit international classique à un droit international contemporain avec l'émergence de nouveaux acteurs sur le plan international ainsi que des mécanismes favorisant leur participation, sans pour autant rompre avec la logique souverainiste fondatrice de cet ordre politico-juridique. Or, les interdépendances engendrées par la globalisation des risques (dont les risques environnementaux), exacerbées par la mondialisation et rappelées récemment par la crise sanitaire de la Covid-19, «ébranlent le dogme de la souveraineté ${ }^{10}$ qui doit se renouveler à la lumière des exigences de coopération et de solidarité. À ces interdépendances, s'ajoute l'universalisme des valeurs. Ils participent, tous les deux, à une réorientation de la souveraineté étatique afin d'intégrer dans la gouvernance mondiale environnementale les objectifs partagés par la communauté internationale (voir, par exemple, les Objectifs du développement durable - ODD). Si la puissance étatique demeure le pivot des relations internationales, la coopération est devenue une obligation internationale générale, et cela parce que les États seuls ne peuvent ni faire face aux risques globaux ni mettre en œuvre leurs valeurs communes.

\section{La propriété}

C'est à cette même époque Moderne que l'usage collectif de biens communs a laissé place à la propriété individuelle. Étymologiquement, la propriété exprime l'appartenance et l'exclusivité ; ce qui est «propre » à une personne ainsi que l'appropriation des biens par l'Homme. L'article 544 du Code civil français la définit comme «le droit de jouir et de disposer des choses de la manière la plus absolue, pourvu que l'on n'en fasse pas un usage prohibé par les lois ou par les règlements ». Cette définition, qui date de 1804, est marquée par son caractère libéral et s'inscrit dans un contexte historique donné. Ayant acquis une valeur constitutionnelle ${ }^{11}$, le droit de propriété occupe une place majeure dans l'ordre juridique français. Or, la Charte de l'environnement, elle aussi dotée d'une valeur constitutionnelle, vient la nuancer. Le droit des biens est alors profondément bouleversé par l'impératif de protection environnementale ${ }^{12}$. Certes, la propriété n'est pas une notion juridique enfermée en elle-même. L'idée qu'elle doit avoir une « fonction sociale » remonte au début du $X X^{e}$ siècle ${ }^{13}$. S'y ajoute désormais une fonction environnementale faisant en sorte que, en y attachant une logique de responsabilité, cette conception nouvelle de la propriété soit en harmonie avec la protection environnementale ${ }^{14}$.

\footnotetext{
${ }^{10}$ DELMAS-MARTY M., "De la souveraineté solitaire à la souveraineté solidaire ", disponible à : http://www.collegium-international.org/index.php/fr/contributions/229-de-la-souverainete-solitaire-a-lasouverainete-solidaire.

${ }^{11}$ Décision du Conseil constitutionnel n॰81-132 du 16 janvier 1982 (loi sur les nationalisations) qui rappelle la valeur constitutionnelle des principes énoncés par la Déclaration de 1789, y compris le caractère fondamental du droit de propriété.

${ }_{12}$ TREBULLE F.-G. (2010), «Environnement et droit des biens », Le droit et l'environnement, Dalloz, Thèmes et commentaires.

${ }^{13}$ DUGUIT L. (1999 [1911]), Les Transformations du droit privé depuis le Code Napoléon, Paris, La Mémoire du droit ; DUGUIT L. (1925 [1913]), Les Transformations du droit public, Paris, A. Colin.

${ }^{14}$ GRIMONPREZ B. (dir.) (2018), Le droit des biens au service de la transition écologique, Paris, Dalloz.
} 
La notion de propriété, replacée dans le contexte de l'entreprise, implique également des transformations ${ }^{15}$. Approcher les entreprises sous l'angle des faisceaux de droits ${ }^{16}$ conduit à revisiter l'idée selon laquelle les actionnaires seraient propriétaires de l'entreprise, à approfondir le rôle de l'entreprise au sein de la société et à proposer une révision de sa définition juridique ${ }^{17}$. La loi française $n^{\circ} 2019-486 \mathrm{du}$ 22 mai 2019 relative à la croissance et la transformation des entreprises, dite loi PACTE, a modifié le Code civil pour élargir l'intérêt social aux enjeux sociaux et environnementaux (art. 1833) et a fait entrer dans ce Code des notions nouvelles comme celles de « raison d'être » ou de « société à mission ». Dans la continuité de ce mouvement de prise en compte des enjeux environnementaux par les entreprises, le Conseil constitutionnel dans sa décision du 31 janvier 2020 ( $n^{\circ} 2019-823$ QPC) considère qu'il n'est pas inconstitutionnel de faire primer la protection de la santé et de l'environnement sur l'exercice de la liberté d'entreprendre.

\section{La personnalité}

La crise écologique remet en cause la place accordée aux entités de la nature, appréhendées traditionnellement par le régime des biens. Si elles font déjà l'objet de protections (délimitation de certaines aires environnementales protégées, par exemple), nous assistons à l'émergence fulgurante de la personnification de la nature, consacrée par différents ordres juridiques lui ayant accordé le statut de sujet de droits. Une telle évolution entraîne comme conséquence pratique la possibilité de la représenter en justice en son nom et dans son intérêt ${ }^{18}$. Or, le résultat est la prolifération de décisions de justice accordant un statut juridique particulier aux éléments de la nature ${ }^{19}$ dans le dessein d'une meilleure protection de l'environnement. Si la France ne connaît pas une telle évolution jurisprudentielle, d'autres pays ont accordé à ces entités la personnalité juridique en vue de leur offrir une protection plus renforcée. Par exemple, en Nouvelle Zélande, la rivière Whanganui a été dotée de la personnalité juridique à travers la reconnaissance par le Parlement de son statut d'entité vivante (15 mars 2017). De même, en Amérique latine, le juge colombien a eu l'occasion de reconnaître la personnalité juridique de la rivière Atrato $^{20}$, puis a déclaré l'Amazonie colombienne sujet de droits en vue de

\footnotetext{
${ }^{15}$ ROBÉ J.P. (1999), L'entreprise et le droit; ROGER B. (dir.) (2012), L'entreprise. Formes de la propriété et responsabilités sociales, Paris, Éd. des Bernardins.

${ }^{16}$ DEAKIN S. (2012), "The Corporation as Commons: Rethinking Property Rights, Governance and Sustainability in the Business Enterprise", Queen's law Journal, 37 (2), pp. 339-381.

${ }^{17}$ RENOUARD C. (2013), Éthique et entreprise, Éditions de l'Atelier; CAPRON M. \& QUAIREL F. (2015), L'entreprise dans la société. Une question politique, La Découverte ; SENART J.-D. \& NOTAT N. (2018), "L'entreprise, objet d'intérêt collectif », rapport des ministres de la Transition écologique et solidaire, de la Justice, de l'Économie et des Finances et du Travail, disponible à : https://www.economie.gouv.fr/mission-entreprise-et-interet-general-rapport-jean-dominique-senardnicole-notat.

${ }^{18}$ SHELTON D. (2015), "Nature as Legal Person", in CAMPROUX DUFFRENE M.-P. \& SOHNLE J., La représentation de la nature devant le juge : approches comparative et prospective, Vertigo, Horssérie 22.

${ }^{19}$ MILON P. (2018), Analyse théorique du statut juridique de la nature, thèse de doctorat, Université Aix-Marseille.

${ }^{20}$ Cour constitutionnelle T-622 de 2016, v. LAFAILLE F. (2019), “Constitution éco-centrique et État social de droit. À propos du constitutionnalisme andin ", Revue française de droit constitutionnel, vol. 118, n², pp. 333-355.
} 
renforcer la protection de cet «écosystème vital pour l'avenir global » ${ }^{21}$. Une transformation plus profonde de l'ordre juridique a vu le jour en Équateur et en Bolivie qui ont, dans leurs constitutions respectives (datées de 2008 et 2012), reconnu à la nature le statut de sujet de droits. Pour ces deux États, l'harmonie et l'équilibre de la vie sont rétablis grâce à l'attribution de droits collectifs et l'instauration d'une cosmovision fondée sur les notions de bien-vivre et d'équité sociale et environnementale entre tous les vivants.

\section{La responsabilité}

Face aux risques écologiques, la responsabilité apparaît comme l'un des principaux instruments de réponse. Elle pèse sur différents acteurs, publics mais aussi privés, et concerne notamment de plus en plus les entreprises ${ }^{22}$. Définie généralement comme le devoir de répondre des effets dommageables d'une action ou d'une inaction, la responsabilité sur le plan juridique suppose l'existence d'une règle de droit dont le non-respect implique une sanction ou l'obligation d'indemniser. Elle suppose l'imputabilité de l'acte. Or, le schéma classique de la responsabilité - dommage, fait générateur, causalité - ne semble pas opérationnel face à des objectifs communs de développement durable qui impliquent, au-delà de la traditionnelle imputation des faits du passé, une orientation plus clairement dirigée vers le futur qui dépasse les conséquences prévisibles ${ }^{23}$. La responsabilité doit ainsi s'adapter en déplaçant le curseur vers la prévention et l'anticipation (responsabilité ex-ante).

Le volet préventif de la responsabilité en matière environnementale est de plus en plus incarné par les notions de responsabilité sociétale de l'entreprise (RSE) et des organisations (RSO). La prolifération de standards internationaux publics et privés convergent vers l'affirmation d'une due diligence (que l'on peut traduire en français par « devoir de vigilance »). La loi française de mars 2017 sur le devoir de vigilance des sociétés mères et donneuses d'ordres a été pionnière et inspire des réformes aux niveaux régional et mondial. Selon cette loi, les entreprises doivent anticiper les risques, les cartographier, prévoir des mesures concrètes pour les éviter (mesures qui intégreront leur plan de vigilance qui devra être publié) et mettre en œuvre cellesci. Ce n'est pas un hasard si le contentieux émergent fondé sur cette loi concerne notamment des questions environnementales. Cette RSE, de plus en plus saisie par le droit, voire par le juge ${ }^{24}$, ne deviendrait-elle pas cette « responsabilité de l'avenir ${ }^{25}$ capable d'accompagner la transition écologique?

\footnotetext{
${ }^{21}$ Cour suprême de Justice, Claudia Andrea Lozano Barragán et al. c. Présidence de la République et al., 5 avril 2018, STC 4360-2018, n¹1001-22-03-000-2018-00319-01, p. 45.

${ }^{22}$ MARTIN-CHENUT K. (2015), "Quelles responsabilités face aux exigences d'une démocratie environnementale? ", in CANAL-FORGUES E. (dir.), Démocratie et diplomatie environnementales : acteurs et processus en droit international, Paris, Pedone, pp. 281-300.

${ }^{23}$ Voir MARTIN-CHENUT K., QUENAUDON R. (dir.) (2016), Développement durable : mutations ou métamorphoses de la responsabilité ?, Paris, Pedone.

${ }^{24}$ MARTIN-CHENUT K. \& QUENAUDON R. (dir.) (2016), La RSE saisie par le droit : perspectives interne et internationale, Paris, Pedone ; CHANTEAU J. P., MARTIN-CHENUT K. \& CAPRON M. (dir.) (2017), Entreprise et responsabilité sociale en question. Savoirs et controverses, Paris, Classiques Garnier, pp. 239-260.

${ }^{25}$ Envisagée en 2004 par THIBIERGE C. (2004), Avenir de la responsabilité et responsabilité de l'avenir, Dalloz, p. 577.
} 